Original scientific paper

\title{
CFD SIMULATION ON HYDRODYNAMICS OF UNDERWATER VEHICLE WITH DUCTED PROPELLERS
}

\author{
Dai, Y.*; Zhang, Y. Y.*; Bian, J. N.*; Han, K. ${ }^{* *}$; Zhu, X. ; Huang, Z. H. ${ }^{* * *} \&$ Xie, Y.** \\ ${ }^{*}$ College of Mechanical and Electrical Engineering, Central South University, Changsha 410083, \\ China \\ ${ }^{* *}$ School of Traffic \& Transportation Engineering, Central South University, Changsha 410083, China \\ ${ }^{* * * *}$ Hunan Institute of Engineering, Xiangtan 411104, China \\ E-Mail: hkun@ @csu.edu.cn ( ${ }^{\#}$ Corresponding author)
}

\begin{abstract}
Underwater vehicles are currently the most effective equipment for exploring ocean mineral resources, whose movement performance been paid more attention by many scholars. According to the underwater vehicle integrating with 19A/Ka4-55 type ducted propellers, this paper researches the hydrodynamic characteristics of the overall vehicle in water under different movement states by leveraging CFD method. Combining structural grids and non-structural grids, an underwater vehicle model with 19A/Ka4-55 ducted propellers is constructed according to the designed propeller arrangement. A specific test rig is set up to verify the accuracy and effectiveness of the CFD method. Finally, the hydrodynamic performance of the integral underwater vehicle carrying the ducted propeller under different working conditions is further analysed. The result suggests that the vehicle still has sufficient propulsion at the inflow velocity of $1 \mathrm{~m} / \mathrm{s}$ in the forward direction. Under ascending condition, the maximum running speed of the operating vehicle is around $0.5 \mathrm{~m} / \mathrm{s}$. It also has shown the feasibility and necessity of hydrodynamic performance analysis.

(Received in June 2021, accepted in August 2021. This paper was with the authors 1 month for 1 revision.)
\end{abstract}

Key Words: Underwater Vehicle, Ducted Propeller, Hydrodynamics Characteristics, CFD Simulation, Test Verification

\section{INTRODUCTION}

As a crossed research realm involving ocean engineering and robotics, the underwater vehicle is an effective equipment for exploring ocean mineral resources. Suffering from insufficient motivation, poor sustainability, and poor ability to resist emergencies and restricting vehicle performance, the underwater vehicle is always the research hotspot of many scholars $[1,2]$. Owing to superior hydrodynamic performance and high work efficiency, the ducted propeller, an improved form of the propeller, has been preferred in modern ships and underwater robots The surrounding catheter is adopted as a drainage device. The restriction of shape aggravates the disturbance of the fluid velocity caused by the rotation of the propeller. The ducted propeller can improve the propulsion performance, accelerate or decelerate the device, and also work as a rudder on small ships. A ducted propeller, for its superior performance characteristics, has been widely used in ocean engineering equipment [3].

Early research on propellers was an appendage and extension of the development of the shipping industry. The design of ducted propellers was firstly proposed and verified to effectively increase the thrust of high-speed heavy ships Subsequently, the method of propeller theory with the potential flow theory was gradually developed. The guiding theories mainly include lifting line theory, lifting surface theory, and panel method $[4,5]$. These methods have contributed to analysing the hydrodynamic performance of propellers, however, regardless of the fluid viscosity, they still have limitations to a certain degree.

Nowadays, depending on the development of computational fluid dynamics (CFD), Reynolds Average Navier-Stokes (RANS) equation used to calculate the viscosity and test verification, has become the main analysis method of hydrodynamic property and motion 
characteristics for conventional propellers [6-9]. These methods apply equally to ducted propellers. In general, the influence of Reynolds number on the torque of ducted propellers is relatively higher and is quite necessary to pursue research on ducted propellers based on the RANS equation [10]. By changing the geometry parameters of the ducted propeller, numerical investigations were carried out to explore the effects of the geometry structure on the hydrodynamic performance with high efficiency, high quality and low cost [11, 12]. Compared traditional propellers and ducted propellers in terms of propulsive force, hydrodynamic properties and geometry parameters, some scholars discussed the structural characteristics, functional advantages and applicable occasions via CFD methods [13, 14]. By leveraging numerical analysis, the geometry structure of ducted propellers and related components could be optimized [15, 16]. Scale effects comparable for a ducted propeller is a useful guide for fullscale design $[10,17]$. Bhattacharyya et al. [18] developed a scaling method for ducted propellers based on CFD method. Some studies have performed numerical and experimental analysis on the vortex and interaction of ducted propellers [19,20].

However, the ducted propeller is inseparable from an underwater operation vehicle. Most of the recent research focus has been on the isolated ducted propeller, not considering the travel performance of the overall underwater vehicle with a ducted propeller. This paper aims to bridge this gap. The CFD method is effectively used to simulate the situation of deep-sea operation vehicles underwater [21,22]. And it is also widely used to analyse the influence of moving objects on the surrounding flow field [23,24]. In this paper, the CFD method was tested and verified, and a simulation model of the overall underwater vehicle is established. The numerical investigation on the hydrodynamic performance of the overall underwater vehicle carrying the 19A+Ka4-55 ducted propeller under different working conditions is conducted.

\section{THEORY AND VERIFICATION}

The hydrodynamic performance of the propeller is related to the interaction force with the surrounding fluid. Varying rotation speed, the mutual influence relationship between different inlet and outlet velocities can be obtained and usually expressed as a dimensionless coefficient to avoids the disturbances of the shape and size of the propeller. The following formulas are the characteristic coefficients of the propeller to reflect its hydrodynamic performance.

Advance coefficient:

$$
J=\frac{v_{A}}{n D}
$$

Propeller thrust coefficient:

$$
K_{T_{P}}=\frac{T_{P}}{\rho n^{2} D^{4}}
$$

Duct thrust coefficient:

$$
K_{T_{D}}=\frac{T_{D}}{\rho n^{2} D^{4}}
$$

Total thrust coefficient:

$$
K_{T}=\frac{T_{P}+T_{D}}{\rho n^{2} D^{4}}
$$

where $T_{p}$ is the propeller thrust, $T_{D}$ is the duct thrust, $T$ denotes the total duct propeller thrust; $n$ is the propeller speed, $D$ is referring to the propeller diameter, $\rho$ represents the water density, $V_{A}$ is the propeller advance speed.

The underwater operation vehicle is subjected to lift due to the pressure difference. However, during low-speed driving, the lift force relative to its own gravity is negligible, so it 
can be regarded as a turbulence problem. The turbulence in the forward and sinking directions of the underwater operating vehicle can be simplified as:

$$
F_{R}=C_{R} \frac{\rho v^{2}}{2} A_{R}
$$

where $C_{R}$ is the total drag coefficient, and $A_{R}$ is the total shape coefficient.

Turbulence of the water appears under the action of the ducted propeller. Proper handling of turbulence is critical to the hydrodynamic analysis of ducted propellers. Viscosity is the basic property of fluid media, and the Reynolds Average Navier-Stokes (RANS) equation adds an additional viscosity term to deal with this issue. The turbulence model contains a common term: turbulent kinetic energy $k$. By defining the parameter turbulent energy dissipation rate $\varepsilon$, the common standard $k-\varepsilon$ model is developed. The RNG $k-\varepsilon$ model (Re-Normalization Group $k-\varepsilon$ ) is based on the standard $k-\varepsilon$ model to improve the viscosity problem of low Reynolds number, mainly to correct the distortion state. Compared with the standard $k-\varepsilon$ model, the accuracy value is generally improved, the vortex in the turbulent flow is considered, and the turbulent viscosity is corrected. Therefore, the RNG $k-\varepsilon$ model is selected for CFD calculation in this paper.

In view of the overall underwater vehicle integrating with the ducted propeller, it requires simultaneous processing of multiple rotating surfaces, so the sliding mesh technique is exploited to address the movement of the propeller blade in water. The computational domain of the flow field needs to be divided into two parts: the internal zone wrapping the propeller blade is set to the rotating region, and the speed is the same as the spindle speed of the propeller; The external zone is set to of the static flow field. An intermediate between the two is used to deliver field data. Considering the complexity of the ducted propeller, the rotating calculation domain is meshed in the unstructured grid. To improve the calculation efficiency, the external zone is divided into a small area and a large area. The small area is a small piece of the static flow field in the periphery of the motion model, and in contact with the duct and operation vehicle, and is also meshed in the unstructured grid. The large area of external areas is meshed in the structured grid. As for the flow of low Reynolds, the local grids of the hub, blade leaf tip, the duct wall, and the operation vehicle model housing are refined.

Difficulty accessing a real underwater operating vehicle, this study uses a representative propeller of a certain ship to build a specific test rig to verify the feasibility and veracity of the CFD method. The propeller is connected to the aluminium alloy fixed mount by a hinge. The propeller is driven via a motor, the speed is adjusted by a $12 \mathrm{~A}$ DC speed regulator, and the generated thrust force is monitor by spring dynamometers fixed on the aluminium alloy mount. The specific test rig is as shown in Fig. 1. The dimension of the water tank is $1.2 \mathrm{~m}$ long, $0.6 \mathrm{~m}$ wide and $0.6 \mathrm{~m}$ high.

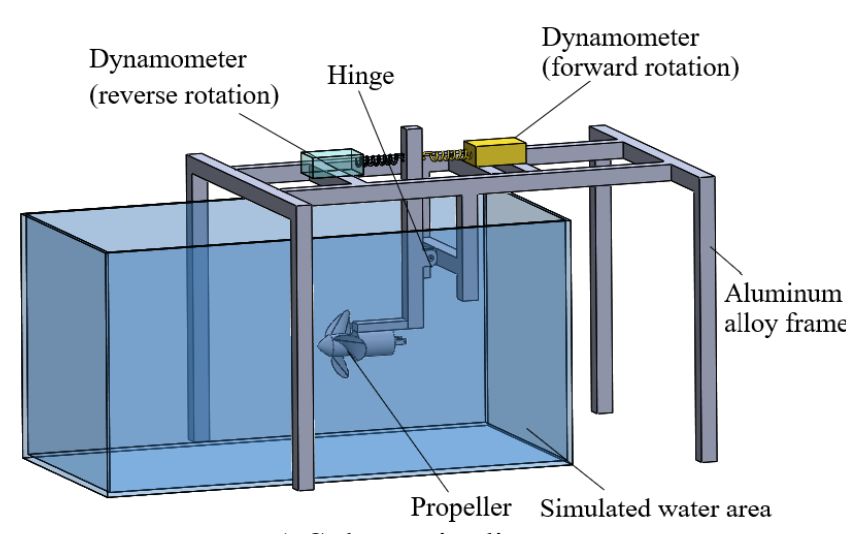

a) Schematic diagram

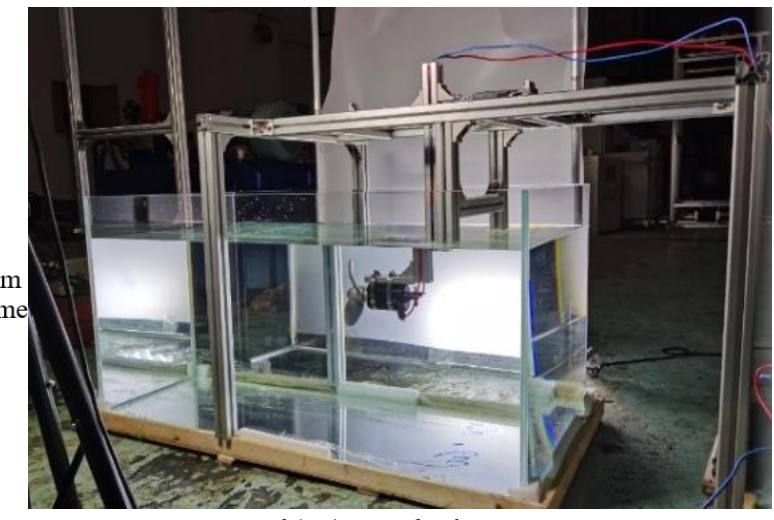

b) Actual photo

Figure 1: Propeller thrust test platform scheme. 
Changing the motor speed, the change laws of thrust versus the propeller speed can be obtained. The motor speed is set to $800 \mathrm{rpm}, 1000 \mathrm{rpm}, 1500 \mathrm{rpm}$, and $2000 \mathrm{rpm}$, respectively. The experimental and numerical thrust of the for-ward-rotating blades and the reverse-rotating blades at different speeds are compared in Fig. 2 below.

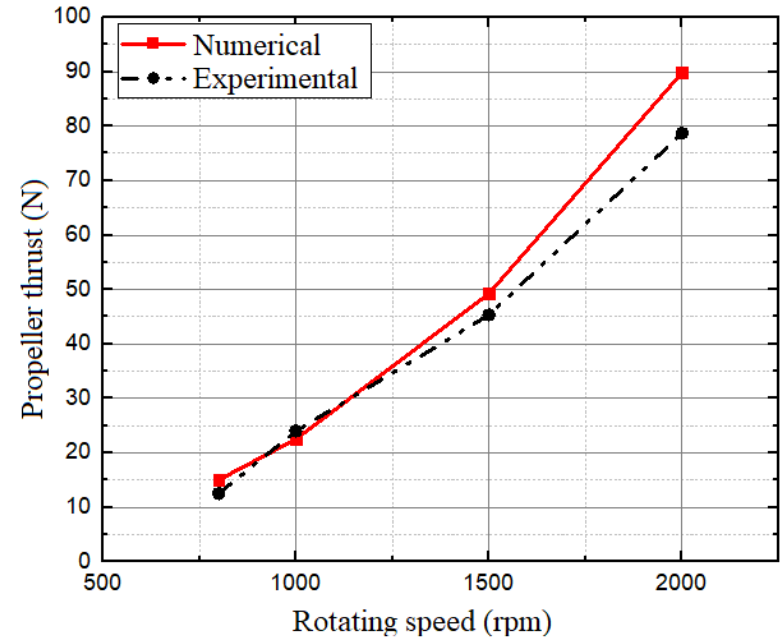

a) Forward rotation

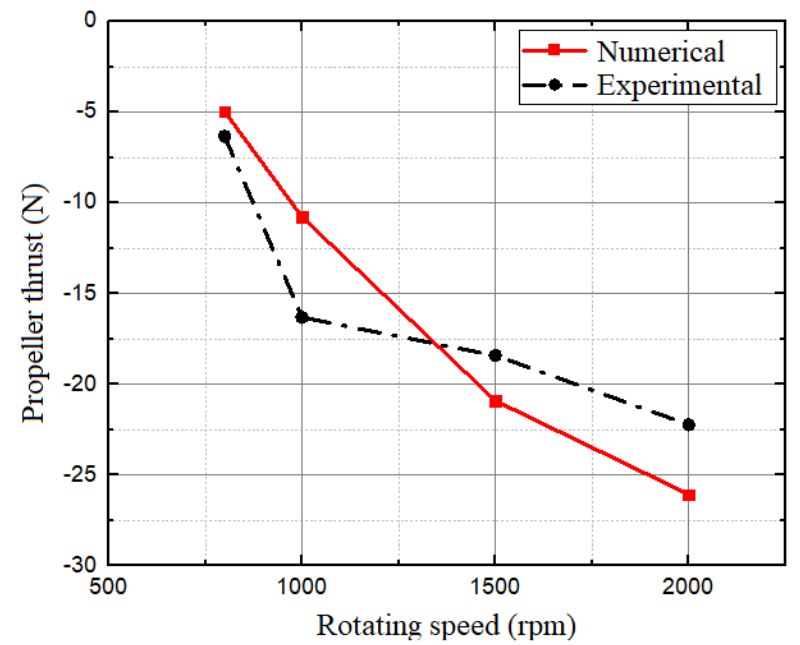

b) Reverse rotation

Figure 2: Change laws of thrust versus the propeller speed.

According to Fig. 2, under the condition of forward rotation, for the experimental and numerical thrust, the change trends are consistent. Both have almost coincided at low speed, and the experimental value is slightly smaller than the numerical result at high speed. When the propeller rotates in the reverse direction, there is a relatively small deviation between the experimental data and the numerical result. As the thrust is relatively small when the propeller is reversed, it is inevitable that partial backflow will occur in the limited test tank, and have a certain influence on the experimental results. In a word, the experiments are basically consistent with the CFD simulations value, it is indicated that the CFD method in this study is reliable and effective.

\section{METHODOLOGY}

\subsection{Propeller layout design}

Underwater operation vehicle should have basic functions of floating and walking in water, and realize various movements such as forward, steering, backward, floating and sinking. As the power source, the propeller's layout directly affects the direction of thrust and operating performance. The underwater operating vehicle needs to be equipped with a variety of operating equipment, requires a relatively large propulsive force. Designed for underwater operations, the operating vehicle can rotate in the state of landing, so the steering performance requirements in water can be appropriately reduced. The selected layout of the propellers is shown in Fig. 3 a, this arrangement can provide sufficient thrust in the main forward direction and heave direction, and have quite good trim and heel performance. The operating speed of the underwater vehicle usually does not exceed $1 \mathrm{~m} / \mathrm{s}$. Therefore, the Ka 4-55 type propeller blade matched with the conventional 19A duct is selected as the ducted propeller. According to the selected propeller layout, the three-dimensional model of the underwater operation vehicle integrating with the ducted propellers is developed as shown in Fig. $3 \mathrm{~b}$, the length, width and height of the model are $1.1 \mathrm{~m}, 0.9 \mathrm{~m}$, and $0.65 \mathrm{~m}$ respectively. 


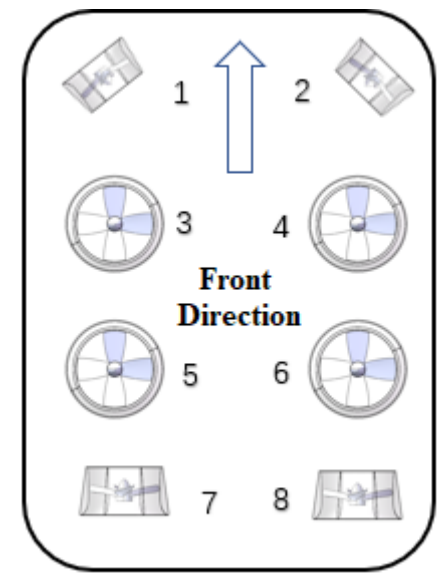

a) Propeller arrangement

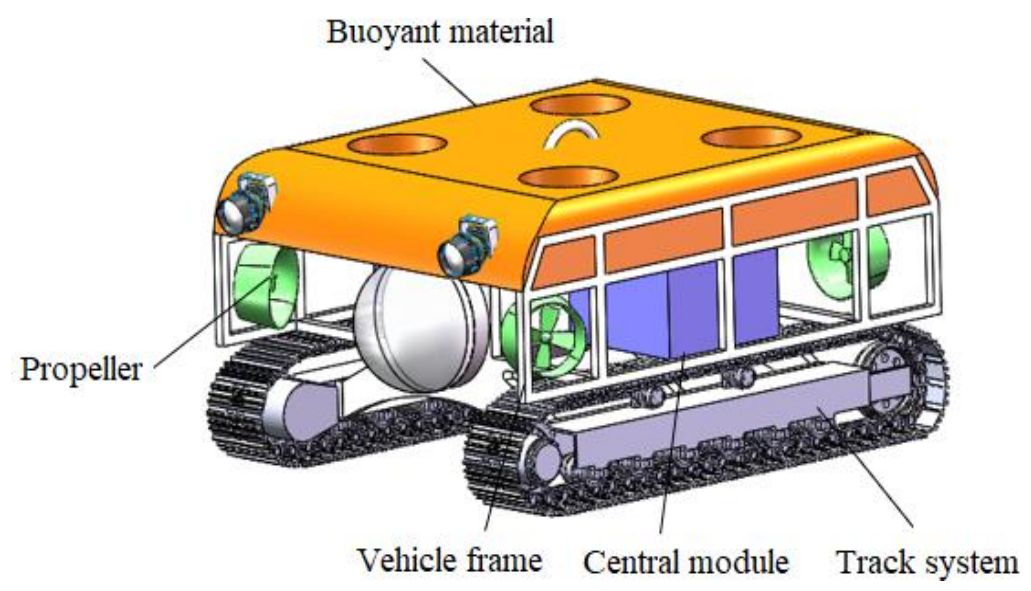

b) Integral underwater vehicle

Figure 3: Schematic diagram of the model.

\subsection{Numerical setup and grids}

Affected by the complex propeller, the main body of the underwater vehicle, and underwater resistance, the hydrodynamic performance of the overall vehicle would be complicated. Moving forward and ascending are common movement states of deep-sea underwater operation vehicles. According to the numerical setup and grid described above, the combination of structured grid and unstructured grid is adopted.

The external region away from the operating vehicle is the static calculation domain, and meshed in the structured grid to reduce the number of grids. The internal computational domain wrapping the vehicle is meshed in the unstructured grid. The rotation of ducted propeller blades is realized by adopting the sliding mesh method, as in section 2 . The grid graph of the vehicle with ducted propellers in the forward condition is shown in Fig. 4 a. The total number of grids is 4.04 million. The average value of the grid skewness is 0.267 , and the maximum value is 0.86 . The grid graph in the ascending condition is shown in Fig. 4 b. The total number of grids is around 4.48 million. The average skewness of the grid is 0.205 , and the maximum skewness is 0.856 .

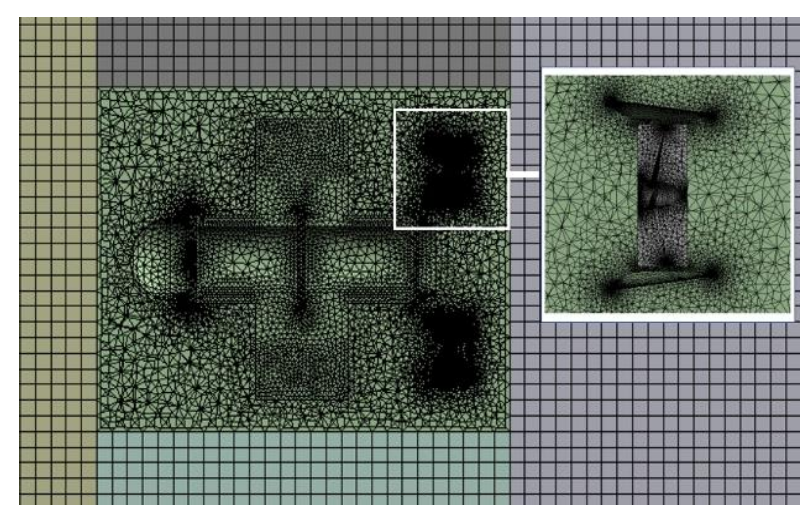

a) Forward condition

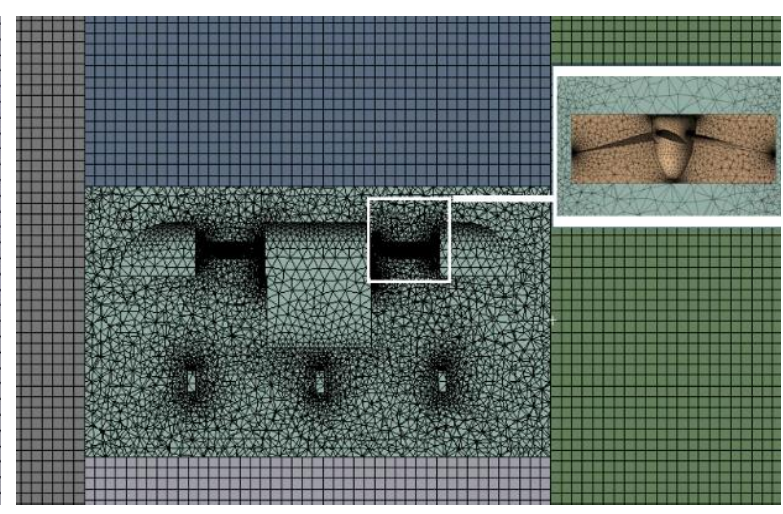

b) Ascending condition

Figure 4: Grid graph of operating vehicle with ducted propellers.

\section{RESULTS AND DISCUSSION}

\subsection{Forward condition}

The underwater operating vehicle is driven by the ducted propeller, the thrust generated is related to its speed co-efficient, and affects the forward and turning speed of the operating 
vehicle, the change of the rotation speed also means the change of the resistance from the surrounding water. Therefore, propeller thrust is an important parameter for evaluating the movement. The thrust generated under different working conditions could affect the hydrodynamic performance of the operating vehicle with the ducted propellers. In this analysis, the change rules of thrust in the steady state versus inflow velocity under the forward condition are obtained via CFD method. The velocity contours of the deep-sea operating vehicle at different inflow velocities are obtained, as shown in Figs. 5, 6, and 7 (i.e. the front view, vertical view, and left view of the velocity contours, respectively.). In the process of moving forward, the resistance produced by the incoming flow has an obvious influence on the interaction between the deep-sea operation vehicle and the surrounding water.
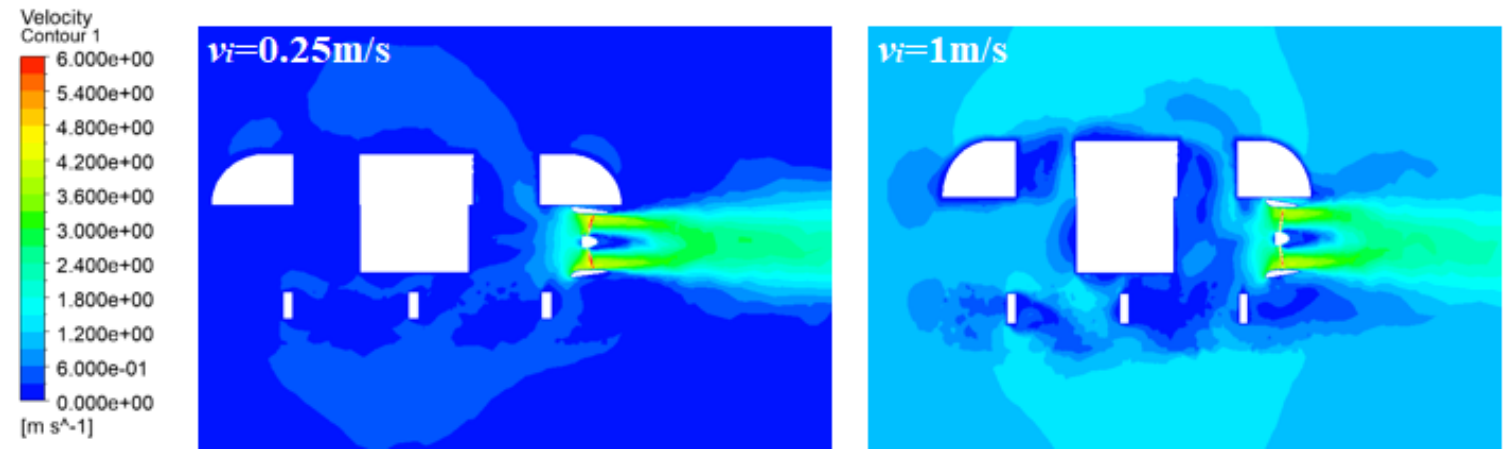

Figure 5: Front view of the velocity contours of the operating vehicle versus inflow velocity.
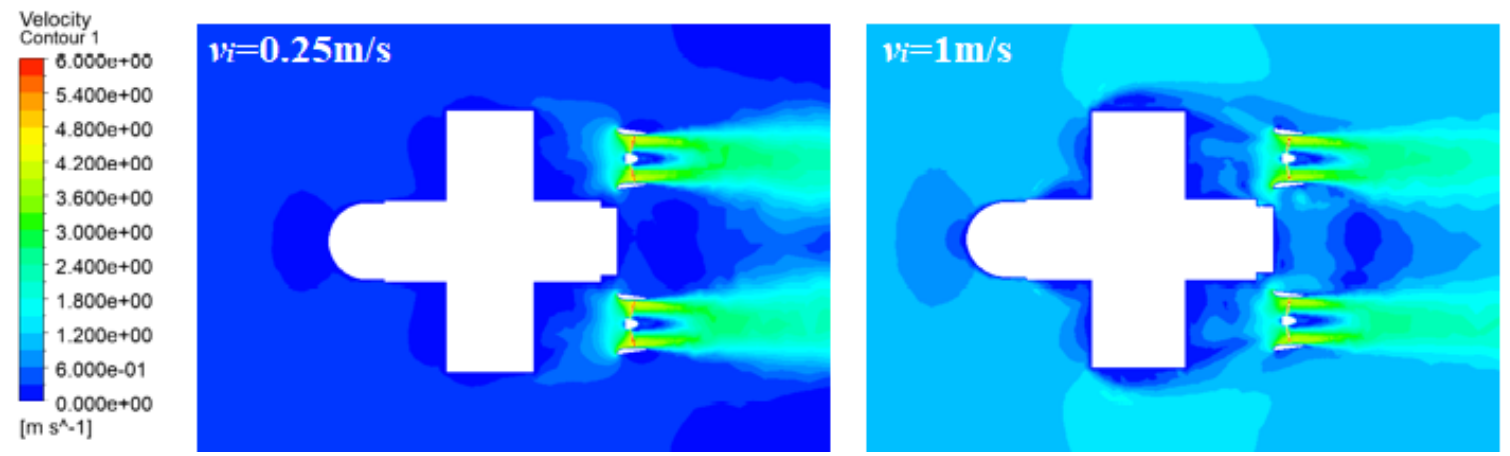

Figure 6: Vertical view of the velocity contours of the operating vehicle versus inflow velocity.
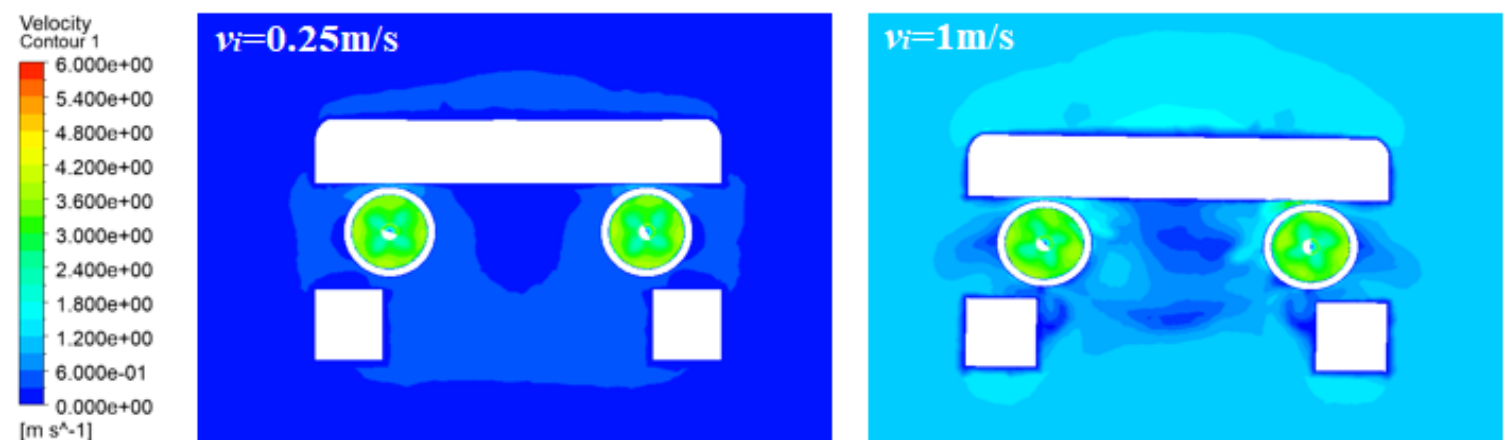

Figure 7: Left view of the velocity contours of the operating vehicle versus inflow velocity.

From the velocity contours, at low inflow velocity, the incoming flow has fewer effects on the fluid field surrounding the underwater vehicle, except for the complex flow around the highspeed rotating propeller. The propeller could pull in low-speed water to accelerate the flow near the entrance, and push out water to re-acceleration at the exit of the propeller along its direction, this shows a good hydrodynamic performance of the ducted propeller. As the inflow velocity is $1 \mathrm{~m} / \mathrm{s}$, the flow field characteristics of the operating vehicle are extremely stronger than that 
of the low speed at $0.5 \mathrm{~m} / \mathrm{s}$, the fluid flow is not only propelled by the propeller, but also significantly impacted by the inflow velocity. As shown in Fig. 5, the propeller accelerates the inhalant water and produces a thrust. As can be seen from Fig. 6, the flow velocity on the back is relatively small due to the flow guiding effect, resulting in a large pressure difference and plenty of water flows in and out through the duct. See Fig. 7, the influencing each other between two propellers is weak at low inflow velocity $0.5 \mathrm{~m} / \mathrm{s}$, whereas the effect has become clearer at $1 \mathrm{~m} / \mathrm{s}$, indicating that two propellers may cause nonuniform flow once the inflow velocity reaches a critical value, so it is necessary to control the maximum travel speed. In addition, it is noted that the flow field near the sharp edges of the operating vehicle is more severe, so the geometry structure of the operating vehicle should be optimized to make it have a better hydrodynamic performance.

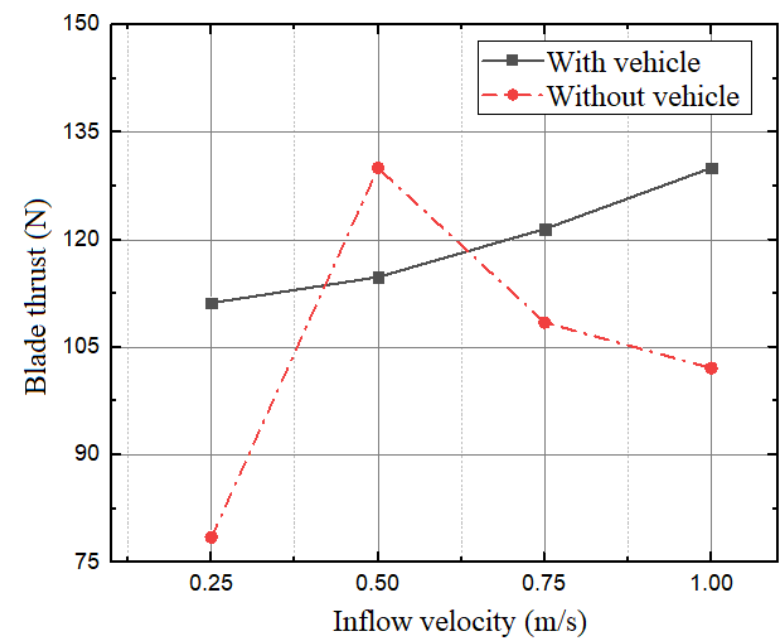

a) Blade thrust

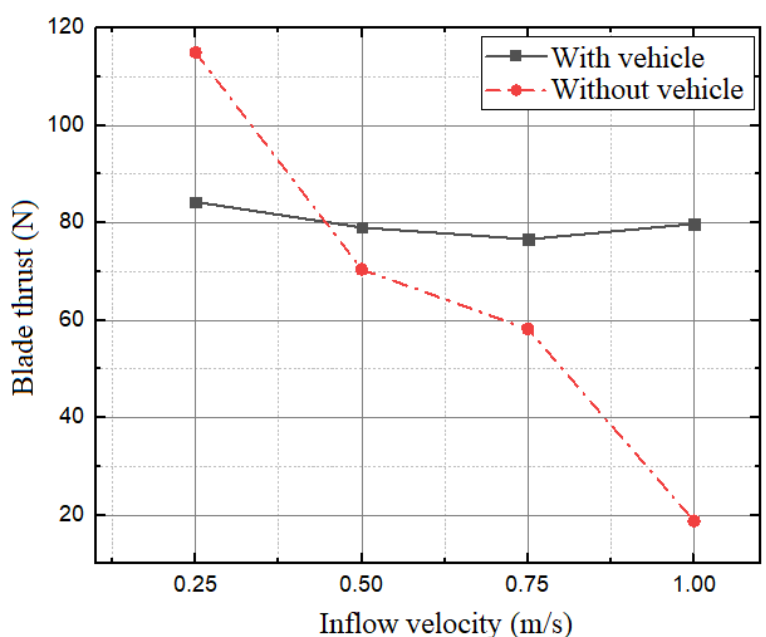

b) Duct thrust

Figure 8: Thrust of ducted propeller versus inflow velocity.

When the rotating speed of the ducted propeller is $2000 \mathrm{rpm}$, Fig. 8 provides the blade thrust and duct thrust with and without the underwater vehicle at different inflow velocities. From Fig. 8 a, the generated blade thrust is low at low inflow velocity, reaches its peak when the velocity is $0.5 \mathrm{~m} / \mathrm{s}$, then gradually decreases, the curve exhibits a parabola relationship. It shows that an appropriate inflow velocity contributes to accelerating the speed rate of water flow through the blades, thereby increasing the blade thrust. Once reaching the peak, increasing the incoming flow will produce resistance. In the case of the operating vehicle, as the inflow velocity is not exceeding $1 \mathrm{~m} / \mathrm{s}$, the vehicle body has a blocking effect on the incoming flow, the peak has not been reached within the range, and the thrust is increasing with the increase of the incoming flow. As for Fig. $8 \mathrm{~b}$, both ducts with and without the underwater vehicle produce great thrust at low inflow velocity, the duct thrust decreases as the inflow velocity increases. The duct thrust without the operating vehicle is greater than that produced with the vehicle body, have the main effect catheter under the condition of flow velocity increasing thrust to slow. Owing to the vehicle body, the deceleration of the duct thrust with the vehicle body is not significant.

Fig. 9 illustrates the change trends of the total thrust of the ducted propeller with and without the operating vehicle. At low inflow velocity, there is a little difference between the two. With the increase of the velocity, the total thrust of the propeller with the operating vehicle is increasing, while that without the vehicle body increases at first and then decreases. At inflow velocity $1 \mathrm{~m} / \mathrm{s}$, the thrust of the ducted propeller without the vehicle body is obviously affected by the incoming flow. It indicates that at low inflow velocity, the propeller mainly generates thrust to overcome the fluid flow movement around itself, but with the increase of the velocity, 
the vehicle body blocks the incoming flow, thereby the propeller is conducive to providing a greater thrust and power for the operation vehicle.

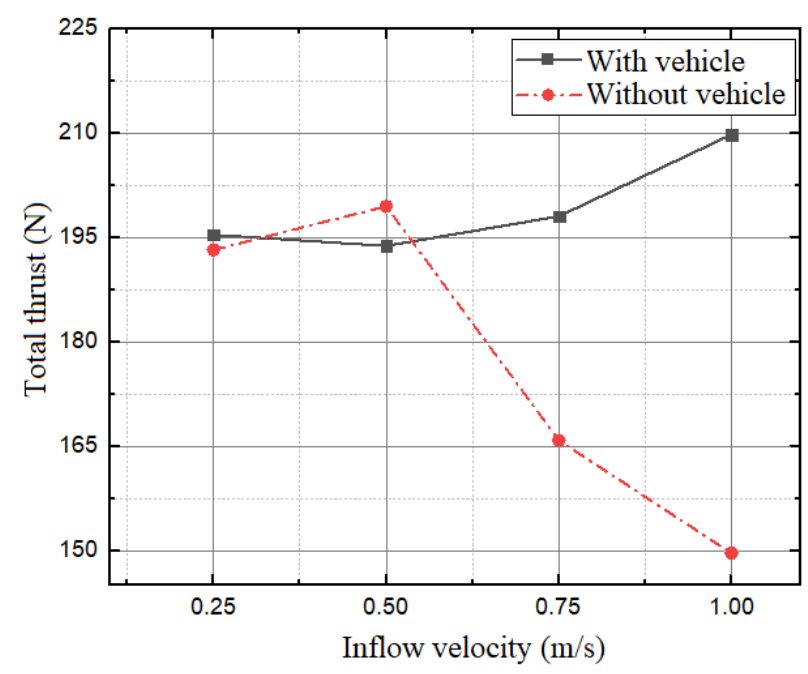

Figure 9: Change trends of the total thrust of the ducted propeller.

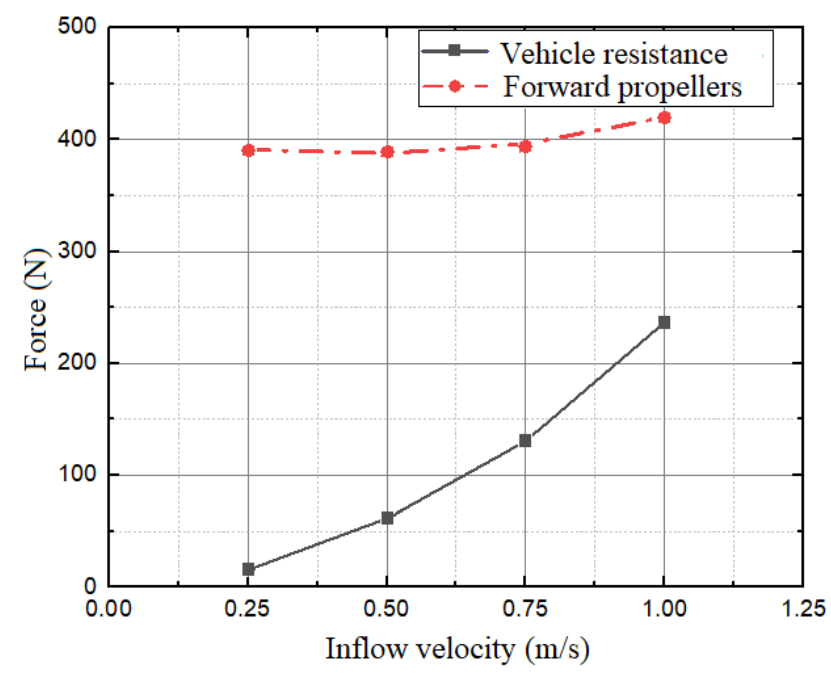

Figure 10: Total thrust and resistance against the running speed.

Moreover, the total thrust produced by two ducted propellers and running resistance from the underwater vehicle in the forward direction at different running speeds are also analysed. As illustrated in Fig. 10, the resistance increases quickly with the running speed, while the total thrust is relatively stable and tends to grow slightly larger at the speed is less than $1 \mathrm{~m} / \mathrm{s}$. The incoming flow could increase its thrust property, and the total thrust is twice the running resistance at the maximum speed, indicating that the running speed can be further increased, and the underwater vehicle has sufficient power in the forward direction.

\subsection{Ascending condition}

The thrust in the upward direction of the underwater vehicle is also one of the critical parameters to measure its hydrodynamic performance and determines whether the vehicle rises or sinks. The weight of the operating vehicle in water has been described in section 3 . To achieve free float and sink without considering the tow rope, the total thrust of the propeller must be greater than the sum of gravity and resistance. In this analysis, with the rotating speed of the propeller is $2000 \mathrm{rpm}$ the change rules of thrust in the steady state versus ascent rate under the ascending condition are obtained via CFD method. The front view and left view of the velocity contours 
of the deep-sea operating vehicle at different ascent velocities are obtained, as shown in Figs. 11 and 12.
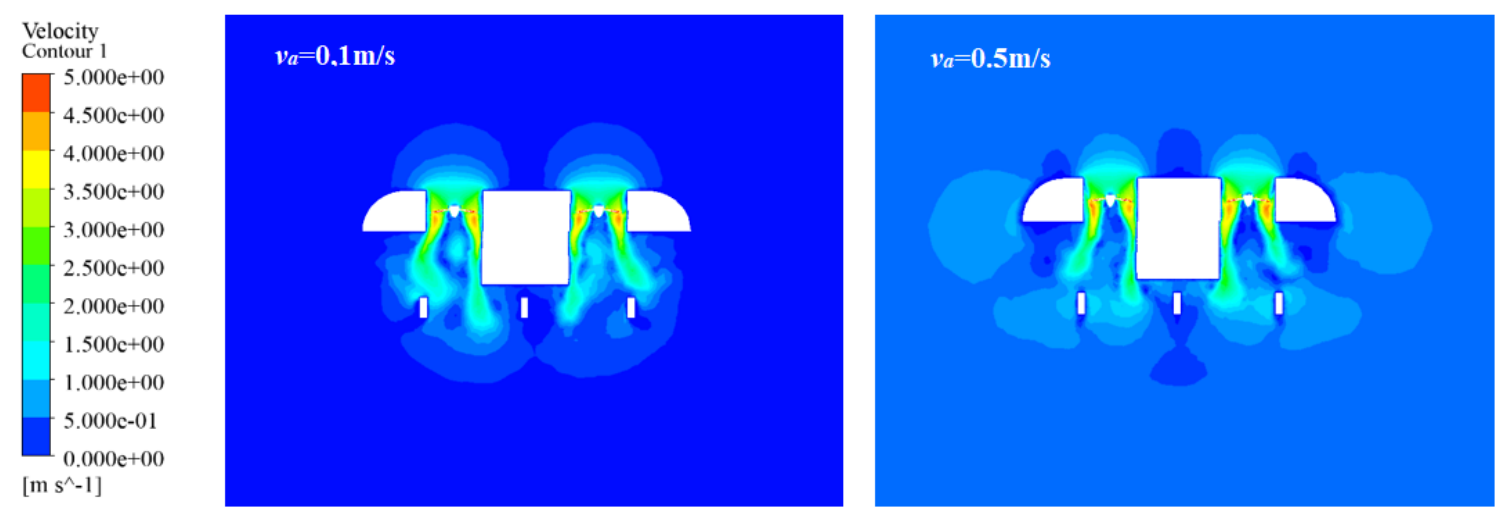

Figure 11: Vertical view of the velocity contours of the operating vehicle versus ascent velocity.
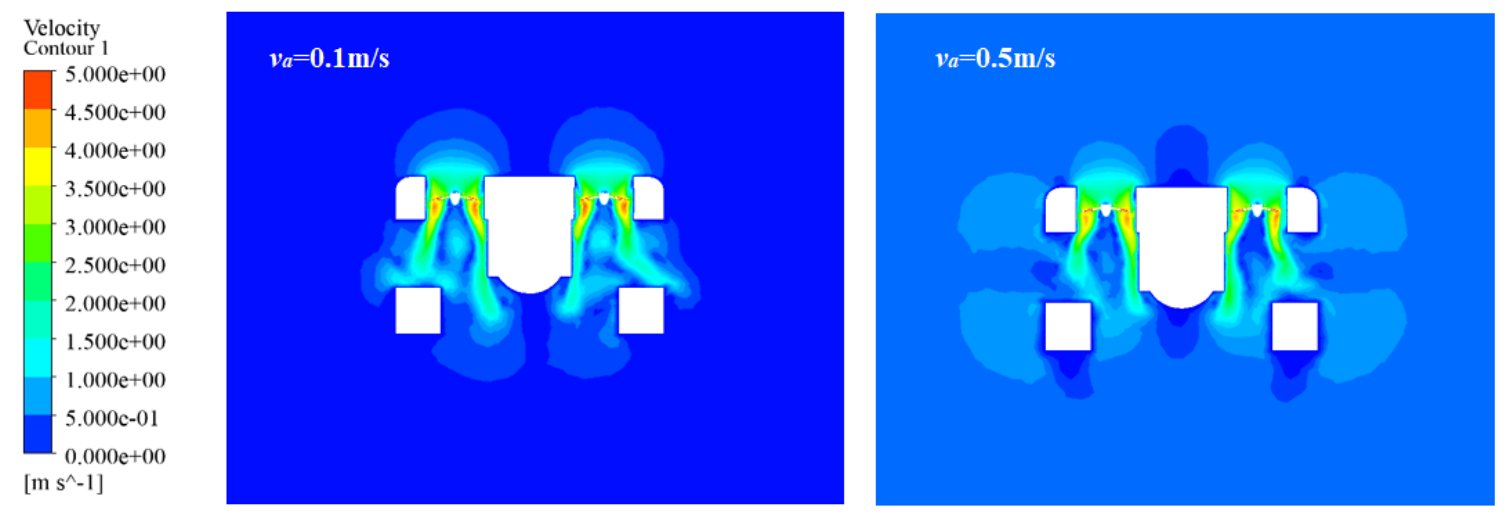

Figure 12: Left view of the velocity contours of the operating vehicle versus ascent velocity.

At a low ascent rate, the fluid flow field is mainly affected by the high-speed rotating propeller. The suction of the water significantly changes the fluid velocity near the upper end. Blocked by the vehicle body, most streamlines in the wakefield of the lower end of the propeller are offset. Its hydrodynamic force has a great influence on the operating vehicle. At a high ascent rate, the overall vehicle is not only affected by the high-speed rotating propellers, but also influenced by the incoming flow, especially at the sharp point. There are some low-speed regions occur under the floating body on the back of the forward direction, under the tracks and under the electronic chamber, this is due to insufficient time for the fluid below flowing in.

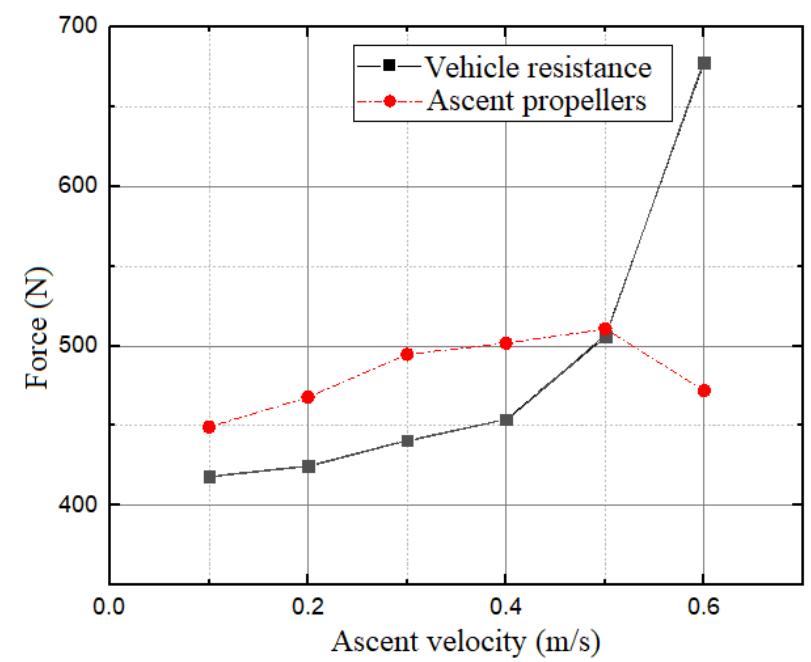

Figure 13: Total thrust and resistance against the running speed under ascending condition. 
Furthermore, the total thrust produced by four ducted propellers and running resistance from the underwater vehicle under ascending condition at different running speeds are also analysed. As shown in Fig. 13, the total resistance of the operating vehicle increases with the speed, and it becomes more apparent when the speed exceeds $0.4 \mathrm{~m} / \mathrm{s}$. The total thrust of the propellers increases slightly as the speed is no more than $0.5 \mathrm{~m} / \mathrm{s}$, while at $0.5 \mathrm{~m} / \mathrm{s}$, the total thrust becomes smaller due to the negative effect of the incoming flow. The horizontal axis of the intersection of the curves is $0.5 \mathrm{~m} / \mathrm{s}$, after this the total resistance of the vehicle gradually increases while the thrust is relatively insufficiency. Those indicate that the maximum floatation speed with the propeller $2000 \mathrm{rpm}$ is $0.5 \mathrm{~m} / \mathrm{s}$.

\section{CONCLUSION}

By leveraging the CFD technique, this paper investigates the hydrodynamic characteristics of the overall underwater vehicle carrying with 19A+Ka4-55 ducted propellers under forward and ascending conditions. The flow field distribution and thrust of the propeller have been analysed, and the vehicle resistance and the total thrust of the propeller are also compared. Moreover, a specific test rig of a common marine propeller has been set up to verify the research method of hydrodynamic performance. The following conclusions are obtained:

Under forward condition, the thrust of the propellers with and without the underwater vehicle at different incoming velocity is compared in this work. Within $1 \mathrm{~m} / \mathrm{s}$, the thrust has not yet reached the peak of the ducted propeller, it is proportionate to the incoming velocity. As the incoming velocity is $1 \mathrm{~m} / \mathrm{s}$, the thrust is 2 times the total resistance.

Under ascending condition, by comparing the total thrust of the propellers with the total resistance in the direction of rising, the maximum rising speed is determined to be about $0.5 \mathrm{~m} / \mathrm{s}$. Meanwhile, it is found that there is a large deviation between the theoretical resistance and the numerical results of the operating vehicle and reflects the feasibility and necessity of hydrodynamic characteristics analysis. Via CFD method, this paper provides theoretical foundations and technical support for the hydrodynamic performance evaluation and design optimization of the underwater vehicle carrying with ducted propellers.

\section{ACKNOWLEDGEMENT}

This research was funded by the National Natural Science Foundation of China (Grant No. 51774324) and the Natural Science Foundation of Hunan Province (Grant No. 2019JJ40380 and 17B063).

\section{REFERENCES}

[1] Saltaren Pazmino, R.; Garcia Cena, C. E.; Alvarez Arocha, C.; Aracil Santonja, R. (2011). Experiences and results from designing and developing a 6 DoF underwater parallel robot, Robotics and Autonomous Systems, Vol. 59, No. 2, 101-112, doi:10.1016/j.robot.2010.10.005

[2] Sahoo, A.; Dwivedy, S. K.; Robi, P. S. (2019). Advancements in the field of autonomous underwater vehicle, Ocean Engineering, Vol. 181, 145-160, doi:10.1016/j.oceaneng.2019.04.011

[3] Takekoshi, Y.; Kawamura, T.; Yamaguchi, H.; Maeda, M.; Ishii, N.; Kimura, K.; Taketani, T.; Fujii, A. (2005). Study on the design of propeller blade sections using the optimization algorithm, Journal of Marine Science and Technology, Vol. 10, No. 2, 70-81, doi:10.1007/s00773-005-0197$\mathrm{y}$

[4] Lerbs, H. W. (1952). Moderately loaded propellers with a finite number of blades and an arbitrary distribution of circulations, Transactions of the Society of Naval Architects and Marine Engineers (SNAME), Vol. 60, 73-123

[5] Yamasaki, H.; Ikehata, M. (1992). Numerical analysis of steady open characteristics of marine propeller by surface vortex lattice method, Journal of the Society of Naval Architects of Japan, Vol. 1992, No. 172, 203-212, doi:10.2534/jjasnaoe1968.1992.172_203 
[6] Castro, A. M.; Carrica, P. M.; Stern, F. (2011). Full scale self-propulsion computations using discretized propeller for the KRISO container ship KCS, Computers \& Fluids, Vol. 51, No. 1, 3547, doi:10.1016/j.compfluid.2011.07.005

[7] Zhang, B.; Ding, C.; Liang, C. (2021). High-order implicit large-eddy simulation of flow over a marine propeller, Computers \& Fluids, Vol. 224, Paper 104967, 18 pages, doi:10.1016/j.compfluid.2021.104967

[8] Villa, D.; Franceschi, A.; Viviani, M. (2020). Numerical analysis of the rudder-propeller interaction, Journal of Marine Science and Engineering, Vol. 8, No. 12, Paper 990, 22 pages, doi:10.3390/jmse8120990

[9] Gao, Z.; Yang, D.; Li, P.; Dong, Y. (2019). Numerical analysis on the effect of artificial ventilated pipe diameter on hydrodynamic performance of a surface-piercing propeller, Journal of Marine Science and Engineering, Vol. 7, No. 8, Paper 240, 18 pages, doi:10.3390/jmse7080240

[10] Abdel-Maksoud, M.; Heinke, H.-J. (2002). Scale effects on ducted propellers, Proceedings of the $24^{\text {th }}$ Symposium on Naval Hydrodynamics, 744-759

[11] Chrusciel, T.; Ciba, E.; Dopke, J. (2014). CFD and FEM model of an underwater vehicle propeller, Polish Maritime Research, Vol. 21, No. 3, 40-45, doi:10.2478/pomr-2014-0028

[12] Zou, J.; Tan, G.; Sun, H.; Xu, J.; Hou, Y. (2020). Numerical simulation of the ducted propeller and application to a semi-submerged vehicle, Polish Maritime Research, Vol. 27, No. 2, 19-29, doi:10.2478/pomr-2020-0023

[13] Andersson, J.; Eslamdoost, A.; Vikstrom, M.; Bensow, R. E. (2018). Energy balance analysis of model-scale vessel with open and ducted propeller configuration, Ocean Engineering, Vol. 167, 369-379, doi:10.1016/j.oceaneng.2018.08.047

[14] Villa, D.; Gaggero, S.; Tani, G.; Viviani, M. (2020). Numerical and experimental comparison of ducted and non-ducted propellers, Journal of Marine Science and Engineering, Vol. 8, No. 4, Paper 257, 21 pages, doi:10.3390/jmse 8040257

[15] Gaggero, S.; Villa, D.; Tani, G.; Viviani, M.; Bertetta, D. (2017). Design of ducted propeller nozzles through a RANSE-based optimization approach, Ocean Engineering, Vol. 145, 444-463, doi:10.1016/j.oceaneng.2017.09.037

[16] Tani, G.; Villa, D.; Gaggero, S.; Viviani, M.; Ausonio, P.; Travi, P.; Bizzarri, G.; Serra, F. (2017). Experimental investigation of pressure pulses and radiated noise for two alternative designs of the propeller of a high-speed craft, Ocean Engineering, Vol. 132, 45-69, doi:10.1016/ j.oceaneng.2017.01.015

[17] Bhattacharyya, A.; Krasilnikov, V.; Steen, S. (2016). Scale effects on open water characteristics of a controllable pitch propeller working within different duct designs, Ocean Engineering, Vol. 112, 226-242, doi:10.1016/j.oceaneng.2015.12.024

[18] Bhattacharyya, A.; Krasilnikov, V.; Steen, S. (2016). A CFD-based scaling approach for ducted propellers, Ocean Engineering, Vol. 123, 116-130, doi:10.1016/j.oceaneng.2016.06.011

[19] Gaggero, S.; Tani, G.; Viviani, M.; Conti, F. (2014). A study on the numerical prediction of propellers cavitating tip vortex, Ocean Engineering, Vol. 92, 137-161, doi:10.1016/ j.oceaneng.2014.09.042

[20] Zhang, Q.; Jaiman, R. K. (2019). Numerical analysis on the wake dynamics of a ducted propeller, Ocean Engineering, Vol. 171, 202-224, doi:10.1016/j.oceaneng.2018.10.031

[21] Dai, Y.; Su, Q.; Zhang, Y. Y. (2020). A new dynamic model and trajectory tracking control strategy for deep ocean mining vehicle, Ocean Engineering, Vol. 216, Paper 108162, 13 pages, doi:10.1016/j.oceaneng.2020.108162

[22] Dai, Y.; Xue, C.; Su, Q.; Huang, X. (2021). Numerical analysis on hydrodynamic characteristics of a deep-sea mining vehicle under three typical motions, Ocean Engineering, Vol. 235, Paper 109446, 14 pages, doi:10.1016/j.oceaneng.2021.109446

[23] Czyz, Z.; Karpinski, P. (2020). Aerodynamic characteristics of the X-tail stabilizer in a hybrid unmanned aircraft, International Journal of Simulation Modelling, Vol. 19, No. 4, 631-642, doi:10.2507/ijsimm19-4-534

[24] Yan, H.; Wang, Y. R.; Shi, H. X.; Li, Q.; Zeng, Y. S.; Jaini, R. (2019). Solid-liquid flow of axial flow pump in loop reactor and operating control with single invert, International Journal of Simulation Modelling, Vol. 18, No. 3, 464-475, doi:10.2507/ijsimm18(3)483 